\title{
Système \\ lymphatique \\ et cerveau
}

> L'activité métabolique considérable du système nerveux central (SNC) requiert un système efficace de drainage et de détoxification tissulaire. Le SNC est pourtant dépourvu de vaisseaux lymphatiques qui assurent le drainage des fluides interstitiels et la surveillance immunitaire dans la plupart des tissus. Un système circulatoire original a été récemment découvert entre le liquide céphalo-rachidien (LCR), le parenchyme cérébral et les vaisseaux lymphatiques des méninges. Le système «glymphatique » implique les espaces périvasculaires et les astrocytes qui drainent les fluides interstitiels depuis et vers le LCR. Des vaisseaux lymphatiques méningés complètent le système glymphatique cérébral en assurant la clairance des macromolécules et des antigènes vers le réseau lymphatique périphérique. Le couple «glymphatique-lymphatiques méningés » constitue ainsi un ensemble de nouvelles cibles thérapeutiques pour améliorer le drainage cérébral et la surveillance immunitaire dans les maladies du SNC. <

\section{Cerveau-système lymphatique : une relation particulière}

Dans un organisme, les cellules entretiennent en permanence des échanges de fluides avec le milieu extracellulaire. Ces échanges sont vitaux, nécessaires à la croissance cellulaire et au bon état des cellules. Dans tous les tissus, les fluides extracellulaires forment un milieu intérieur, dont l'homéostasie doit être maintenue. Cette règle de stabilité nécessite un renouvellement permanent de ce milieu intérieur, sans cesse modifié par l'activité des cellules. Les fluides interstitiels sont donc drainés, ce qui permet de moduler localement leur volume et ainsi d'éviter le gonflement, ou œdème, des tissus provoqué par une rétention d'eau et d'électrolytes. Le drainage du milieu intérieur permet également sa clairance, c'est-

Vignette (Photo (c) Inserm - Caroline Ciumas).

\section{Jean-Léon Thomas ${ }^{1,2}$, Laurent Jacob ${ }^{1}$, Ligia Boisserand ${ }^{2}$}

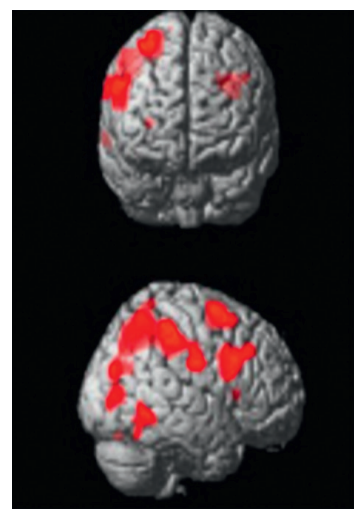

${ }^{1}$ UMRS1127, Sorbonne Université, Institut du cerveau et de la moelle épinière, 7, boulevard de l'Hôpital, 75013 Paris, France. ${ }^{2}$ Department of neurology, Yale University School of Medicine, New Haven, CT, 06511, ÉtatsUnis.

jean-leon.thomas@

icm-institute.org

laurent.jacob@icm-institute.org ligia.boisserand@yale.edu

à-dire l'évacuation des macromolécules et des substances toxiques produites par les cellules.

Dans la plupart des organes, le drainage tissulaire est assuré par le système lymphatique [1]. Le dysfonctionnement des vaisseaux lymphatiques provoque des œdèmes, éventuellement chroniques, ou lymphœdèmes, très invalidants. Les vaisseaux lymphatiques et les ganglions qui y sont associés sont également des voies de propagation et des sites d'expansion des cellules tumorales métastatiques. Le système vasculaire lymphatique est un réseau de vaisseaux endothéliaux dont les extrémités capillaires, en contact avec le milieu intérieur (extracellulaire), sont aveugles et se raccordent par un ensemble de tubes collecteurs à la circulation veineuse. Le plus important de ces tubes collecteurs est le canal lymphatique thoracique qui se déverse dans la veine sous-clavière gauche. Les capillaires lymphatiques sont dénués de revêtement musculaire lisse. Ils sont dotés de jonctions entre les cellules endothéliales dites «en bouton » qui facilitent la recapture de macromolécules et des cellules circulantes du milieu intérieur [2]. Les capillaires lymphatiques drainent le milieu extracellulaire et capturent le mileu intérieur sous forme de lymphe, un fluide circulant dans des vaisseaux, mais dépourvu de globules rouges. La présence de valves et d'une tunique musculaire dans les vaisseaux lymphatiques collecteurs permet de créer un flux unidirectionnel, vers les ganglions lymphatiques et la circulation générale. Les vaisseaux lymphatiques ont une autre fonction essentielle : la surveillance immunitaire des tissus, en assurant la remise en circulation des cellules immunitaires qui patrouillent dans l'espace extracellulaire. Ainsi, les cellules macrophagiques glanent les débris cellulaires et les potentiels antigènes avant de rejoindre la lymphe dans laquelle ils seront transportés vers les ganglions lymphatiques. C'est au niveau de ces noeuds du système lymphatique que se transmet l'information entre cellules macrophagiques et lymphocytes; celle-ci pourra éventuellement activer 


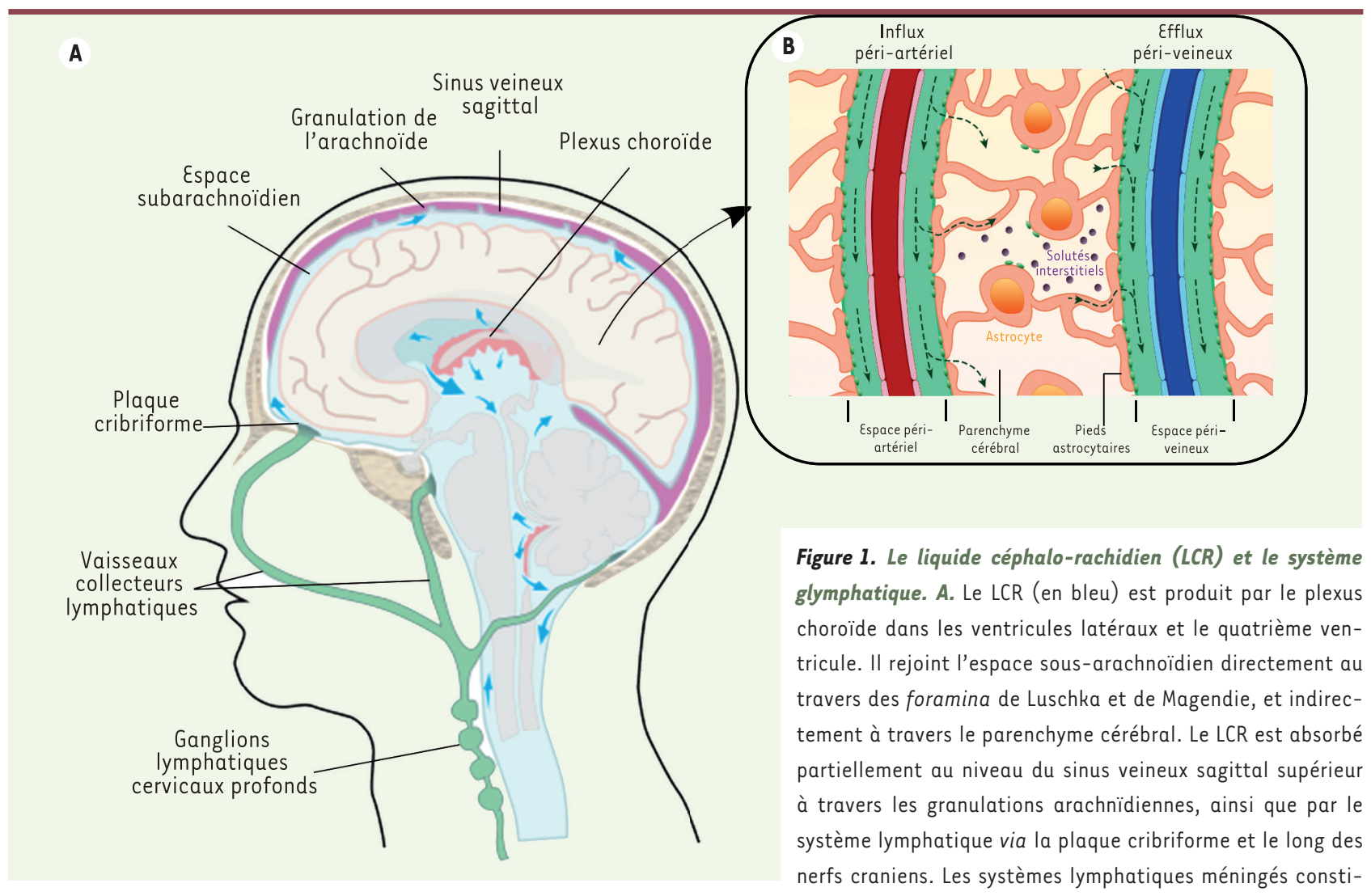

tueraient une autre voie de réabsoption du LCR (inspiré de [5]). B. Circulation des fluides cérébraux à travers le système glymphatique. Un afflux de LCR provenant de l'espace sous-arachnoïdien circule le long des espaces péri-artériels et -artériolaires. Au niveau des capillaires, les fluides périvasculaires sont transférés dans le parenchyme cérébral où leur circulation est dépendante des astrocytes et rejoint les espaces péri-veineux. Un efflux de liquide interstitiel serait drainé dans les espaces péri-veineux et rejoindrait l'espace sous-arachnoïdien (schéma adapté de [16]).

la maturation et l'expansion des clones lymphocytaires spécifiques de l'antigène présenté par les macrophages [3]. Le système lymphatique est donc essentiel à la veille et la défense immunitaires.

Le système nerveux central (SNC), qui correspond à l'encéphale (c'està-dire le cerveau, le cervelet et le tronc cérébral) et la moelle épinière, ne représente qu'environ $2 \%$ du poids du corps, alors qu'il consomme plus de $25 \%$ de l'énergie produite par l'organisme. Cette activité métabolique intense nécessite un système de drainage et de clairance des fluides interstitiels particulièrement efficace. Le SNC ne suit pas la règle génerale du drainage lymphatique de proximité. Son parenchyme est en effet dépourvu de vaisseaux lymphatiques. Comment, alors, le SNC assure-t-il donc cette fonction remplie, dans la plupart des tissus, par le système lymphatique? Et comment peut-il assurer sa surveillance immunitaire en l'absence de vaisseaux lymphatiques, alors que, dans les conditions physiologiques, sa barrière hémato-encéphalique est imperméable aux cellules immunitaires périphériques?

\section{Compartimentation des fluides du SNC}

Le SNC présente une architecture telle que les fluides extracellulaires y sont compartimentés. II s'agit d'un organe creux, drainé au cœur de son axe rostro-caudal ${ }^{1}$ par un important volume de fluide, le liquide céphalo-rachidien (LCR), qui circule dans les quatre ventricules cérébraux et dans le canal de l'épendyme. Le LCR est produit par le plexus choroïde à partir du sang, à une vitesse assez comparable entre les espèces $(0,2$ à $0,6 \mu \mathrm{l} / \mathrm{min} / \mathrm{g}$ de plexus choroïde). Sa production (300 à $1000 \mathrm{ml} / \mathrm{jour}$ chez l'homme adulte) est sous le contrôle de la pression artérielle, du rythme cardiaque et du rythme circadien. Sa composition ionique et protéique est différente de celle du plasma avec davantage d'ions $\mathrm{Na}^{+}$et $\mathrm{Cl}^{-}$et moins d'ions $\mathrm{Ca}^{2+}$ et $\mathrm{K}^{+}$, de protéines, de glucose et d'acides-aminés [4]. En raison de sa faible concentration en molécules organiques, le LCR est considéré comme un réservoir collecteur des métabolites et des déchets produits par le SNC, selon un gradient de concentration décroissant. Le LCR irrigue également la surface externe du SNC et ses enveloppes les plus internes (ou leptoméninges),

${ }^{1}$ L'axe rostro-caudal est perpendiculaire aux plans transverses. Il est parallèle à la colonne vertébrale chez les Vertébrés. 


\section{GLOSSAIRE}

Gradient de concentration : variation continue du nombre de molécules en solution ou dans un gaz, d'un compartiment (cellulaire, tissulaire) où elles sont en forte concentration, vers un autre compartiment où elles sont en moindre concentration.

Trous de Luschka : ouvertures latérales du IVe ventricule permettant le passage du liquide céphalorachidien vers l'espace sub-arachnoïdien. Trou de Magendie : ouverture médiane du IVe ventricule permettant le passage du liquide céphalorachidien vers l'espace sub-arachnoïdien. Neuropile: ensemble du réseau des axones, dendrites, synapses et filaments gliaux du SNC, à l'exclusion des corps cellulaires.

Polygone de Willis : cercle artériel situé à la base du cerveau qui met en relation les différentes artères arrivant au cerveau, à savoir les artères carotides et le système vertébro-basilaire. Ce système d'anastomose permet au cerveau de recevoir du sang, même si l'une des artères du cou est sténosée, c'est-à-dire de diamètre rétréci.

http://www.info-radiologie.ch/polygone-willis.php

Imagerie par résonance magnétique (IRM) : I'IRM du corps humain est fondée sur les propriétés magnétiques de ses atomes d'hydrogène. Le noyau d'hydrogène est un proton (chargé positivement) qui est en mouvement et peut être assimilé à un petit aimant. Lorsque le patient est placé à l'intérieur du tunnel de la machine à IRM, il subit un champ magnétique dans lequel tous ses «protons-aimants » vont s'aligner. Afin de perturber l'équilibre de cet alignement, on produit une excitation par émission d'ondes radio. À une fréquence particulière, les protons se mettent en état de « résonance », c'est-à-dire effectuent un mouvement de rotation. L'arrêt de la stimulation provoque un retour du proton à son état d'alignement normal, ce qui détermine une variation du champ magnétique. Cette variation du champ magnétique est détectable par une antenne et induit la formation d'un courant électrique : c'est le signal IRM.

Séquence IRM, pondération T1 et T2: le temps de répétition (TR) est l'intervalle de temps entre deux excitations. Le temps d'écho (TE) est l'intervalle de temps entre l'excitation et la survenue du signal IRM. Une séquence IRM est un ensemble d'impulsions excitatrices dont les paramètres (TE, TR par exemple) sont ajustés pour obtenir des images ayant un contraste donné (Tl ou T2 par exemple). Dans une image pondérée en $\mathrm{Tl}$, la graisse apparaît hyperintense (couleur claire) et l'eau hypointense. Dans une image pondérée en T2, l'eau apparaît hyperintense (couleur claire) et la graisse un peu plus sombre que l'eau. L'information diagnostique est tirée des différences d'intensité des signaux émis par le tissu normal et le tissu pathologique.

https://www.info-radiologie.ch/resonance_magnetique.php Imagerie par résonance magnétique (IRM) par tenseur de diffusion : technique d'IRM qui permet la cartographie in vivo de la microstructure et de l'organisation des tissus. Elle permet de détecter et quantifier des anomalies de la substance blanche non détectables en imagerie conventionnelle.

qu'il rejoint depuis les ventricules latéraux et le troisième ventricule à travers les trous de Luschka et de Magendie ${ }^{2}$. Les fluides interstitiels cérébraux et spinaux sont donc entourés d'un vaste flux périphérique

${ }^{2}$ Voir Glossaire. de LCR (Figure 1A) [5]. Comment sont-ils drainés? Quelles relations ont-ils avec le LCR ? Le système vasculaire lymphatique contribue-t-il à leur dynamique? Des découvertes récentes permettent de donner des réponses à ces questions et de proposer une modélisation de la dynamique des fluides du SNC.

\section{Dynamique des fluides intestitiels du SNC : le système glymphatique}

Une voie majeure de drainage et de clairance du SNC est constituée par les espaces paravasculaires [6,7], appelés aussi périvasculaires [8]. II s'agit des espaces compris entre la paroi externe des vaisseaux sanguins cérébraux et la membrane basale du tissu nerveux environnant. En dehors des différences sémantiques et des désaccords qui peuvent subsister sur la nature anatomique précise de la bordure de ces espaces, également nommés espaces de Virchow-Robin pour les gros vaisseaux, ils constituent les sites clefs d'entrée des macromolécules issues du LCR dans le parenchyme du SNC. L'existence d'une relation entre LCR et liquide interstitiel cérébral $[9$, 10], et l'entrée du LCR dans les espaces paravasculaires [11, 12] étaient dejà connues lorsqu'en 2012, l'équipe de Maiken Nedergaard a montré, en temps réel chez la souris adulte, le lien entre le flux intracrânien du LCR et la circulation des fluides interstitiels cérébraux [6]. Ces auteurs ont réalisé des injections de traceurs (dextran et ovalbumine) fluorescents dans le LCR des leptoméninges (dans la grande citerne, sous le cervelet), suivies soit d'une imagerie in vivo en temps réel, réalisée avec un microscope biphotonique à travers une fenêtre optique transcrânienne, soit d'une analyse ex vivo par microscopie confocale. Ils ont ainsi pu révéler une voie de circulation paravasculaire depuis le LCR à travers le parenchyme cérébral. L'influx (le débit entrant) de LCR s'effectue le long des espaces péri-artériels et suit un déplacement global, dit de convection. Cette observation a été confirmée in vivo par des études d'imagerie par résonance magnétique (IRM) par tenseur de diffusion, réalisées chez le rat, qui montrent un point d'entrée principal du LCR à la base du crâne, au niveau du polygone de Willis, dans l'espace perivasculaire entourant les artères cérébrales moyennes [13]. Chez l'homme, elle a été confirmée après injection dans le LCR de gadobutrol, un produit de contraste, et analyse des séquences pondérées en $\mathrm{Tl}$ sur une longue période de temps (1, 2, 28 jours) [14]. Une voie d'entrée potentielle du LCR dans les espaces paravasculaires impliquerait des pores spécialisés, ou stomates, qui sont décrits en microscopie électronique à balayage dans l'adventice bordant les vaisseaux des leptoméninges [15]. 
Les astrocytes constituent un des composants cellulaires de la barrière hémato-encéphalique ( $B H E$ ) qui limite les échanges entre le sang et le tissu nerveux. Ils entourent les vaisseaux sanguins de leur prolongements, ou pieds astrocytaires, qui sont riches en transporteurs et canaux ioniques. Le rôle des astrocytes dans le contrôle des fluides du SNC ne se limite cependant pas à faire barrière au flux de solutés provenant de la circulation générale. Ils participent également à la circulation des fluides, depuis les espaces paravasculaires vers et à travers le parenchyme cérébral. Cette contribution au drainage des fluides intestitiels a été mise en évidence pour la première fois par J.J. Iliff et al. [6] qui ont proposé le concept de «système glymphatique» pour signifier que la fonction de drainage «lymphatique » du SNC était assurée par la glie, et non pas par les vaisseaux (Figure 1B) [16]. L'eau et des composants hydrophiliques et lipophiles seraient transportés par convection, à travers et autour du réseau astroglial, depuis les espaces périartériels. L'efficacité de ce système, qui repose sur la pulsatilité des artérioles cérébrales et sur la pression du LCR, est accrue lors du sommeil. C'est en effet durant la période de sommeil que l'activité des neurones baisse, la taille des cellules se réduit et l'espace interstitiel augmente (de $60 \%$ ) [17]. L'activité du système glymphatique dépend aussi de la position du corps [18] et de l'âge de l'individu, le vieillissement s'accompagnant d'une réduction du drainage interstiel cérébral [19]. La contribution de l'aquaporine-4 (AQP4), un canal hydrique astrocytaire, a été proposée [20], mais elle reste discutée $[4,21]$. Le modèle glymphatique défini initialement paraît devoir étre reajusté si l'on considère que le flux de convection du LCR se combine à une diffusion passive, vers le neuropile (l'espace extracellulaire du SNC) [22].

Dans le modèle du système glymphatique, les fluides interstitiels circuleraient des espaces périartériels vers les espaces périveineux, en suivant un gradient de pression décroissante. L'efflux de ces fluides se déverserait ensuite dans le LCR en empruntant l'espace sous-arachnoïdien. Il est donc nécessaire que le LCR soit, lui-même, renouvelé... mais comment est-il évacué de l'espace intracrânien? Dans différentes espèces de vertébrés, plusieurs voies d'évacuation sont connues: les granulations arachnoidiennes, qui sont localisées le long du sinus veineux sagittal supérieur [23, 24]; la plaque cribriforme olfactive, par les canaux lymphatiques de la muqueuse nasale; et le long des gaines des nerfs crâniens, via les vaisseaux lymphatiques qui les longent $[25,26]$. L'importance fonctionnelle de ces voies doit cependant faire l'objet d'une nouvelle évaluation. En effet, l'existence d'un réseau de vaisseaux lymphatiques intracrâniens, dont le rôle dans la recapture intracérébrale des macromolécules et des antigènes, ainsi que dans la circulation des cellules immunitaires, est désormais établi.

\section{Le système lymphatique méningé}

La présence de vaisseaux lymphatiques à proximité du cerveau est suspectée depuis la fin XVIII siècle [27] et, déjà, leur localisation autour des nerfs crâniens avait été identifiée [28]. L'extension du réseau lymphatique au niveau des méninges crâniennes et leur implication dans la circulation des fluides du SNC et des cellules immunitaires sont, en revanche, des observations récentes. Elles ont été publiées chez le rongueur par deux équipes, indépendamment et presque simultanément: la première équipe recherchait l'une des voies d'entrée et de sortie des lymphocytes dans les méninges [29] ; la seconde examinait le circuit drainant le LCR vers les ganglions lymphatiques cervicaux [30]. Ce réseau a depuis été identifié chez d'autres vertébrés, notamment chez le ouistiti (marmouset) [31, 32], et chez I'homme, par l'étude post mortem de méninges [29,31], et aussi in vivo, par IRM [31].

Les cellules partipant au réseau vasculaire lymphatique méningé expriment une combinaison de marqueurs (Proxl/ VEGFR3/LYVE-1/Podoplanin/CCL21)3 ${ }^{3}$ considérée comme étant spécifique de l'endothélium lymphatique [33]. Le développement de modèles de souris transgéniques possédant des gènes codant certaines de ces molécules couplés à des rapporteurs fluorescents a considérablement facilité l'étude in vivo des vaisseaux lymphatiques et de leur localisation à la surface profonde de la boîte crânienne, sur la dure-mère, sans contact direct avec l'encéphale. Ils sont en fait alignés le long des sinus veineux, qui collectent les veines cérébrales, mais aussi le long des artères méningées (Figure 2A, B) [34]. Les cellules immunitaires, notamment les lymphocytes $T$, sont particulièrement abondantes au voisinage des lymphatiques méningés dans lesquels elles circulent [29] (Figure 2D). Néanmoins, la position précise de ces vaisseaux dans la dure-mère, et en particulier la localisation de leur contact direct, s'ils existent, avec le LCR de l'espace sous-arachnoïdien, reste débattue: on ignore encore si les vaisseaux lymphatiques méningés réabsorbent le LCR directement ou si un mécanisme intermédiaire est impliqué [5].

Les vaisseaux lymphatiques méningés drainent les fluides du SNC, les macromolécules et les cellules immunitaires qu'ils contiennent, vers des ganglions lymphatiques cervicaux profonds. L'ensemble de ce circuit peut être suivi, depuis le LCR [29] ou le parenchyme cérébral [30], grâce à l'injection de différents marqueurs colorés ou fluorescents (de 3 à $10^{3} \mathrm{kDa}$ ), dont l'accumulation dans les ganglions peut être réduite expérimentalement, chez des animaux dont le circuit a été génétiquement altéré [30, 32] ou chirugicalement interrompu [29]. La ligature des vaisseaux lymphatiques provenant des ganglions cervicaux profonds accroît l'accumulation de la population de lymphocytes $T$ et de traceurs, du LCR dans les ganglions et dans ses vaisseaux afférents à la base du crâne, ainsi que dans les méninges [29, 32]. La recapture des macromolécules présentes dans le LCR s'effectuerait dans des zones spécialisées du réseau lymphatique méningé (hot-spots) [35] (Figure 2B, C), ce qui pourrait limiter

\footnotetext{
${ }_{3}^{3}$ Prospero homeobox 1 / vascular endothelial growth factor receptor 3 / lymphatic
} vessel endothelial hyaluronan receptor-1/Podoplanin/ C-C motif chemokine ligand 21. 

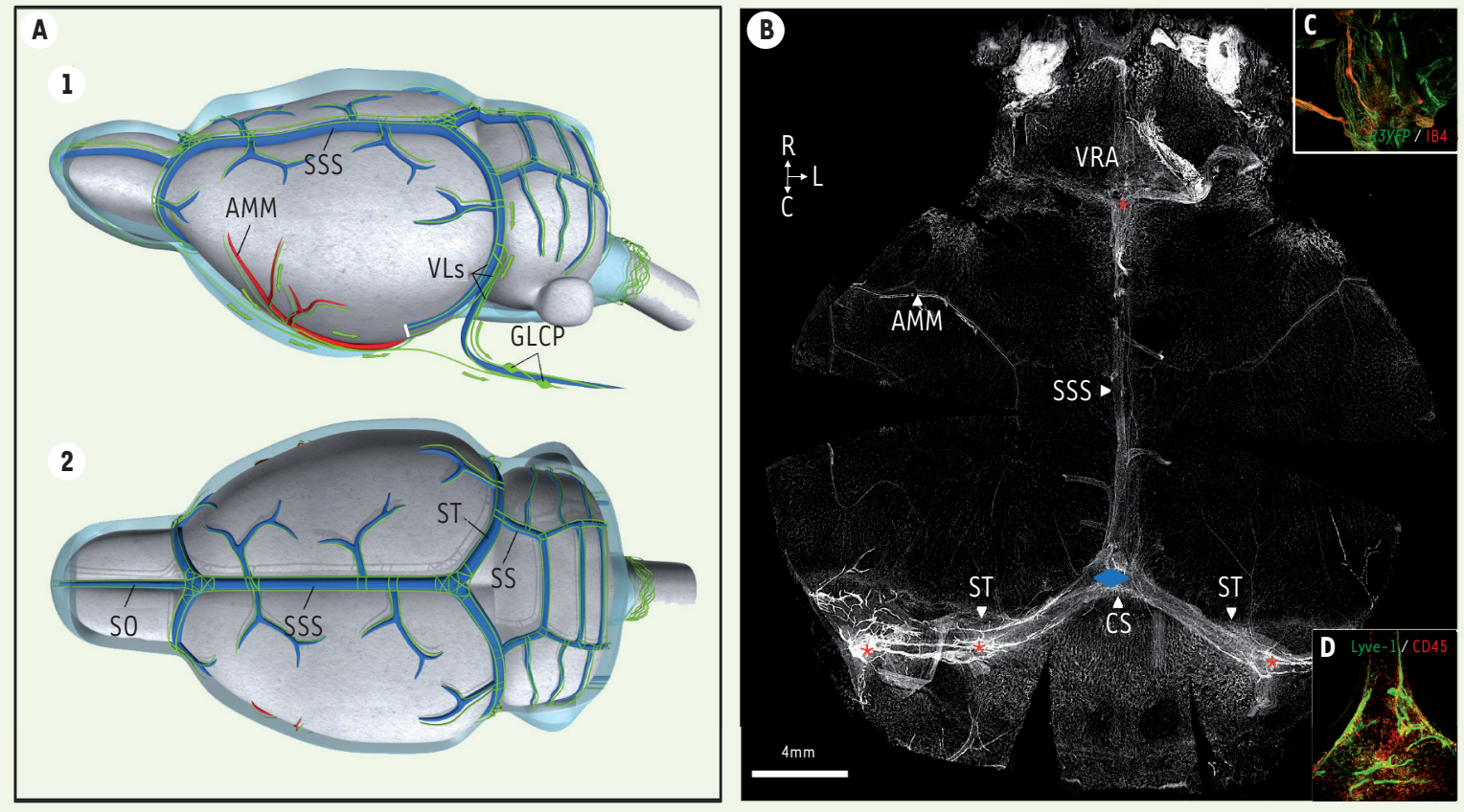

Figure 2. Réseau vasculaire lymphatique méningé de la boîte crânienne. A. Représentation schématique du réseau des vaisseaux lymphatiques méningés autour du cerveau de la souris adulte. 1. Vue latérale montrant les sinus veineux (en bleu : sinus supérieur sagittal [SSS], et sinus transverse $[\mathrm{ST}]$ ), ainsi que les vaisseaux lymphatiques (en vert) qui sont adjacents aux sinus veineux et à l'artère méningée moyenne (MMA) (en rouge). Les vaisseaux lymphatiques drainent la lymphe depuis la dure-mère vers les ganglions lymphatiques cervicaux profonds (GLCP). 2. Vue de dessus du réseau lymphatique méningé associé aux veines durales superficielles, au sinus olfactif (SO) et au sinus sigmoïde (SS) (Schémas adaptés de [34]). B. Face interne d'une boîte crânienne de souris transgénique (VEGFR3YFP) exprimant la protéine fluorescente verte YFP (yellow fluorescent protein) comme rapporteur de l'expression du récepteur VEGFR-3, caractéristique de l'endothélium lymphatique. Les têtes de flèches blanches montrent la localisation des vaisseaux lymphatiques ; les étoiles rouges indiquent les hot-spots du réseau lymphatique spécialisés dans la recapture des macromolécules apportées par l'efflux du système glymphatique ; le losange bleu indique la position du panneau $\mathbf{D}$. $\boldsymbol{C}$. Illustration d'un hot-spot de recapture de macromolécules drainées par les fluides cérébraux, localisé au niveau de la VRA. La macromolécule est ici une lectine fluorescente rouge ( $2 \mu \mathrm{l}$ d'isolectine B4 [IB4 Alexa FluorTM 594 Conjugate; 114 kDa]) injectée dans le LCR de la grande citerne et détectée après 6 minutes dans les vaisseaux lymphatiques méningés (jaune) au niveau de la VRA. D. Concentration de cellules immunitaires (CD45 , en rouge) au voisinage des vaisseaux lymphatiques méningés (Lyve- $\mathrm{I}^{+}$, en vert), au niveau de la région CS (losange bleu en B). VEGFR-3: vascular endothelial growth factor receptor 3 ; CS : confluence des sinus ; SSS : sinus supérieur sagittal ; VRA : veine nasale antérieure ; C : caudal ; L : latéral ; R : rostral.

la contamination par les efflux du système glymphatique. Les vaisseaux lymphatiques méningés participent donc à la clairance du LCR et des fluides interstitiels cérébraux. Ils ne régulent cependant pas leur volume car, en l'absence de vaisseaux lymphatiques méningés, le poids sec du cerveau n'est en effet pas modifié [1]. La recirculation des lymphocytes T via les vaisseaux lymphatiques méningés a été établie en conditions physiologiques [29]. L'importance de cette recirculation pour la surveillance et la réponse immunitaire cérébrale reste en cours d'étude, dans le contexte de plusieurs maladies du SNC, comme nous le verrons plus loin.

\section{Plasticité et implication du lymphatique méningé dans les maladies du SNC}

Les vaisseaux lymphatiques méningés apparaissent à la naissance, tardivement dans le développement du système lymphatique [32].
Au cours du viellissement, le diamètre des vaisseaux se réduit [35]. Chez l'adulte, leur croissance et leur maintien dépendent du facteur lymphangiogénique VEGF-C (vascular endothelial growth factor-C) et de son récepteur VEGFR3 (vascular endothelial growth factor receptor 3 ) qui régissent la phase embryonnaire de la lymphangiogenèse [36]. Des modèles animaux dans lesquels cette signalisation peut-être stimulée ou inhibée, ont été développés par transgénèse ou par pharmacologie, par injection de protéines recombinantes $[29,30,32,35]$. Ces modèles ont permis de montrer que le réseau lymphatique méningé était doué d'une remarquable plasticité, en comparaison de la majorité du réseau vasculaire qui reste stable chez l'adulte. La couverture lymphatique méningée peut 
en effet s'accroître chez l'adulte [32]. Les vaisseaux lymphatiques méningés peuvent aussi se restaurer chez la souris âgée, améliorant le drainage du LCR, la clairance, par exemple, de la protéine bêtaamyloïde impliquée dans la maladie d'Alzheimer, et les performances cognitives [35]. Le VEGF-C apparaît ainsi comme un facteur capable de maintenir l'homéostasie du tissu cérébral au cours du veillissement. Dans le contexte de la maladie d'Alzheimer, l'implication physiopathologique du drainage lymphatique cérébral dans la clairance de la protéine bêta-amyloïde a été montrée par plusieurs études chez la souris, par ligature des ganglions lymphatiques cervicaux [37], ou par traitement avec du VEGF-C pour accroître la couverture lymphatique méningée $[35,38]$. L'effet bénéfique du VEGF-C sur le déclin cognitif est cependant controversé. II reste en effet à établir, avec des protocoles expérimentaux qui soient comparables [35, 38]. Le bénéfice d'un gain de fonction du système lymphatique cérébral n'est par ailleurs pas assuré pour toutes les pathologies du SNC. Ainsi, dans le cas de maladies auto-immunes, comme dans le modèle murin de la sclérose en plaques (encéphalomyélite auto-immune expérimentale ou $\varepsilon A \varepsilon$ ), la réduction du vaisseau lymphatique méningé s'accompagne paradoxalement d'une diminution de la paralysie des souris, qui traduit une diminution de la démyélinisation [39]. Dans ce contexte précis, le vaisseau lymphatique méningé favoriserait la réponse auto-immune des lymphocytes Th (T helper), soit localement, soit en recrutant des cellules dendritiques présentatrices des antigènes de myéline qui les transporteraient vers les ganglions lymphatiques. Les cibles thérapeutiques potentielles sont ici la cytokine CCL2l produite par les vaisseaux lymphatiques méningés, et son récepteur CCR7 ( $C-C$ chemokine receptor type 7), exprimé par les lymphocytes. D'autres modèles de pathologies du SNC sont actuellement étudiés pour examiner le rôle physiopathologique et le potentiel thérapeutique du système lymphatique cérébral. Ces études devraient permettre de caractériser le profil moléculaire des cellules endothéliales lymphatiques et des cellules immunitaires qui leur sont associées, dans les différents contextes pathologiques. Elles devraient également permettre d'identifier de nouveaux bio-marqueurs et des cibles thérapeutiques, liés au système lymphatique cérébral.

En plus du système de drainage glymphatique-lymphatiques méningés, l'encéphale contient des cellules de l'immunité innée : des cellules dendritiques, d'origine périphérique, et des cellules microgliales résidentes, qui contribuent à la clairance et la détoxification du tissu cérébral [40]. Chez le poisson-zèbre, de nouvelles populations de cellules endothéliales lymphatiques, dites murales, isolées et ne formant pas de vaisseaux, ont été identifiées dans les espaces périvasculaires cérébraux. Elles recyclent les déchets des fluides tissulaires dans leur compartiments intracellulaires [41]. Ces cellules présentent des analogies avec les cellules décrites par Mato et al. chez les mammifères, qui sont localisées le long des vaisseaux sanguins méningés [42]. L'intégration fonctionnelle de ces populations mobiles des espaces périvasculaires avec le circuit lymphatique méningé reste cependant à établir.

L'importance des découvertes récentes relatives à la circulation des fluides et des molécules antigéniques dans le SNC, à leur impact sur sa maintenance fonctionnelle, nous rappelle le pouvoir fascinant qu'ont longtemps exercé les fluides cérébraux, considérés comme le siège de l'âme et de l'esprit, de la liquor vitalis évoquée par Galenus de Pergamon (130-201 AD) et par Shakespeare [4]. Nous sommes maintenant à l'épreuve des faits qui devraient bientôt nous éclairer sur le potentiel thérapeutique du système de clairance encéphalique. $\diamond$

\section{SUMMARY}

Lymphatic system in central nervous system

The considerable metabolic activity of the central nervous system (CNS) requires an efficient system of tissue drainage and detoxification. The CNS is however devoid of lymphatic vessels, a vasculature ensuring interstitial fluid drainage and immune survey in other organs. A unique system of drainage has recently been identified between the cerebrospinal fluid (CSF), brain interstitial fluids and meningeal lymphatic vessels. This system is coupling a cerebral "glymphatic" flow with a meningeal lymphatic vasculature. The "glymphatic" system includes perivascular spaces and astrocytes, and drains interstitial fluids, from and towards the CSF. Meningeal lymphatic vessels are functionally linked to the cerebral "glymphatic" efflux by clearing intracerebral macromolecules and antigens towards the peripheral lymphatic system. The "glymphatic"-"meningeal lymphatics" system is potentially offering new therapeutic targets to improve cerebral drainage and immune survey in human CNS diseases. $\diamond$

\section{REMERCIEMENTS}

Les illustrations ont été réalisées par Jean-Mickaël Thomas (Figure 1), Laurent Jacob et Ligia Boisserand (Figure 2). Merci à Hélène Benveniste (Yale) et Elena Nikanorova pour leur aide au design de la Figure 2, à Charles Duyckaerts et Bernard Zalc (ICM) pour leur relecture attentive et leurs commentaires, et à la FRC (Fondation pour la Recherche sur le Cerveau), l'ARC (Association pour la Recherche sur le Cancer) et l'ANR2017 Brainwash pour leurs supports financiers.

\section{LIENS D'INTÉRÊT}

Les auteurs déclarent n'avoir aucun lien d'intérêt concernant les données publiées dans cet article.

\section{RÉFÉRENCES}

1. Aspelund A, Robciuc MR, Karaman S, et al. Lymphatic system in cardiovascular medicine. Circ Res 2016 ; 118 : 515-30.

2. Baluk P, Fuxe J, Hashizume $H$, et al. Functionally specialized junctions between endothelial cells of lymphatic vessels. J Exp Med 2007 ; 204 : 234962.

3. Iwasaki A, Medzhitov R. Control of adaptive immunity by the innate immune system. Nat Immunol $2015 ; 16: 343-53$.

4. Abbott NJ, Pizzo ME, Preston JE, et al. The role of brain barriers in fluid movement in the CNS: is there a "glymphatic" system? Acta Neuropathol 2018 ; 135 : 387-407.

5. Raper D, Louveau A, Kipnis J. How do meningeal lymphatic vessels drain the CNS? Trends Neurosci 2016 ; 39 : 581-6. 


\section{RÉFÉRENCES}

6. Iliff JJ, Wang M, Liao Y, et al. A paravascular pathway facilitates CSF flow through the brain parenchyma and the clearance of interstitial solutes, including amyloid $\beta$. Sci Transl Med 2012 ; 4: 147rall1.

7. Bedussi B, Almasian M, Vos J de, et al. Paravascular spaces at the brain surface: Low resistance pathways for cerebrospinal fluid flow. J Cereb Blood Flow Metab 2018 ; 38 : 719-26.

8. Lochhead JJ, Wolak DJ, Pizzo ME, et al. Rapid transport within cerebral perivascular spaces underlies widespread tracer distribution in the brain after intranasal administration. J Cereb Blood Flow Metab $2015 ; 35$ : 371-81.

9. Cserr HF, Ostrach LH. Bulk flow of interstitial fluid after intracranial injection of blue dextran 2000. Exp Neurol $1974 ; 45: 50-60$.

10. Bradbury MW, Cserr HF, Westrop RJ. Drainage of cerebral interstitial fluid into deep cervical lymph of the rabbit. Am J Physiol $1981 ; 240:$ F329-36.

11. Rennels ML, Gregory TF, Blaumanis OR, et al. Evidence for a paravascular fluid circulation in the mammalian central nervous system, provided by the rapid distribution of tracer protein throughout the brain from the subarachnoid space. Brain Res $1985 ; 326: 47-63$.

12. Rennels ML, Blaumanis $O R$, Grady PA. Rapid solute transport throughout the brain via paravascular fluid pathways. Adv Neurol $1990 ; 52: 431-9$.

13. Harrison IF, Siow B, Akilo AB, et al. Non-invasive imaging of CSF-mediated brain clearance pathways via assessment of perivascular fluid movement with diffusion tensor MRI. Elife 2018 ; 7 : pii e34028.

14. Ringstad G, Valnes LM, Dale AM, et al. Brain-wide glymphatic enhancement and clearance in humans assessed with MRI. JCl Insight $2018 ; 3$ : el21537.

15. Pizzo ME, Wolak DJ, Kumar NN, et al. Intrathecal antibody distribution in the rat brain: surface diffusion, perivascular transport and osmotic enhancement of delivery.J Physiol (Lond) 2018 ; $596: 445-75$.

16. Louveau A, Plog BA, Antila S, et al. Understanding the functions and relationships of the glymphatic system and meningeal lymphatics. J Clin Invest 2017 ; $127: 3210-9$.

17. Xie L, Kang H, Xu Q, et al. Sleep drives metabolite clearance from the adult brain. Science 2013; $342: 373-7$

18. Lee $\mathrm{H}, \mathrm{Xie} \mathrm{L}, \mathrm{Yu}_{\mathrm{u}} \mathrm{M}$, et al. The effect of body posture on brain glymphatic transport. J Neurosci $2015 ; 35: 11034-44$

19. Kress BT, Iliff JJ, Xia M, et al. Impairment of paravascular clearance pathways in the aging brain. Ann Neurol $2014 ; 76: 845-61$.

20. Iliff JJ, Goldman SA, Nedergaard M. Clearing the mind: Implications of dural lymphatic vessels for brain function. Lancet Neurol $2015 ; 14: 977-9$.

21. Smith AJ, Yao X, Dix JA, et al. Test of the glymphatic hypothesis demonstrates diffusive and aquaporin-4-independent solute transport in rodent brain parenchyma. Elife 2017 ; 6 : pii e27679.

22. Asgari M, Zélicourt $D$ de, Kurtcuoglu V. Glymphatic solute transport does not require bulk flow. Sci Rep $2016 ; 6: 38635$.

23. Weed LH. Studies on cerebro-spinal fluid. No. III : the pathways of escape from the Subarachnoid Spaces with particular reference to the Arachnoid Villi. J Med Res 1914 ; 31 : 51-91.

24. Go KG, Houthoff HJ, Hartsuiker J, et al. Fluid secretion in arachnoid cysts as a clue to cerebrospinal fluid absorption at the arachnoid granulation. J Neurosurg $1986 ; 65: 642-8$.

25. Johnston M, Zakharov A, Papaiconomou C, et al. Evidence of connections between cerebrospinal fluid and nasal lymphatic vessels in humans, non-human primates and other mammalian species. Cerebrospinal Fluid Res $2004 ; 1: 2$
26. $\mathrm{Ma} \mathrm{O}$, Ineichen BV, Detmar $\mathrm{M}$, et al. Outflow of cerebrospinal fluid is predominantly through lymphatic vessels and is reduced in aged mice. Nat Commun $2017 ; 8: 1434$

27. Lukić IK, Gluncić V, Ivkić G, et al. Virtual dissection: a lesson from the 18th century. Lancet $2003 ; 362: 2110-3$.

28. Andres KH, Düring $M$ von, Muszynski K, et al. Nerve fibres and their terminals of the dura mater encephali of the rat. Anat Embryol 1987 ; $175: 289-301$.

29. Louveau A, Smirnov I, Keyes TJ, et al. Structural and functional features of central nervous system lymphatic vessels. Nature $2015 ; 523: 337-41$.

30. Aspelund A, Antila S, Proulx ST, et al. A dural lymphatic vascular system that drains brain interstitial fluid and macromolecules. J Exp Med 2015 ; 212 : 991-9

31. Absinta M, Ha SK, Nair G, et al. Human and nonhuman primate meninges harbor lymphatic vessels that can be visualized noninvasively by MRI. Elife $2017 ; 6:$ e29738.

32. Antila S, Karaman S, Nurmi H, et al. Development and plasticity of meningeal lymphatic vessels. J Exp Med 2017 ; 214 : 3645-67.

33. Petrova TV, Koh GY. Organ-specific lymphatic vasculature: From development to pathophysiology. J Exp Med 2018 ; 215 : 35-49.

34. Benveniste $H$, Liu X, Koundal S, et al. The glymphatic system and waste clearance with brain aging: A review. Gerontology 2018 ; 1-14.

35. Da Mesquita S, Louveau A, Vaccari A, et al. Functional aspects of meningeal lymphatics in ageing and Alzheimer's disease. Nature $2018 ; 560: 185-91$.

36. Sáinz-Jaspeado M, Claesson-Welsh L. Cytokines regulating lymphangiogenesis. Curr Opin Immunol 2018 ; $53: 58-63$.

37. Wang L, Zhang $Y$, Zhao $Y$, et al. Deep cervical lymph node ligation aggravates AD-like pathology of APP/PS1 mice. Brain Pathol 2018 ; Sep 7. doi: 10.1111/ bpa.12656.

38. Wen YR, Yang JH, Wang X, et al. Induced dural lymphangiogenesis facilities soluble amyloid-beta clearance from brain in a transgenic mouse model of Alzheimer's disease. Neural Regen Res 2018 ; 13 : 709-16.

39. Louveau A, Herz J, Alme MN, et al. CNS lymphatic drainage and neuroinflammation are regulated by meningeal lymphatic vasculature. Nat Neurosci $2018 ; 21$ : 1380-91.

40. Prinz M, Erny D, Hagemeyer N. Ontogeny and homeostasis of CNS myeloid cells. Nat Immunol $2017 ; 18: 385-92$.

41. Bower NI, Koltowska K, Pichol-Thievend C, et al. Mural lymphatic endothelial cells regulate meningeal angiogenesis in the zebrafish. Nat Neurosci 2017 ; $20: 774-83$.

42. Mato M, Ookawara S, Sakamoto A, et al. Involvement of specific macrophage-lineage cells surrounding arterioles in barrier and scavenger function in brain cortex. Proc Natl Acad Sci USA 1996; 93 : 3269-74.

TIRÉS À PART

L. Jacob

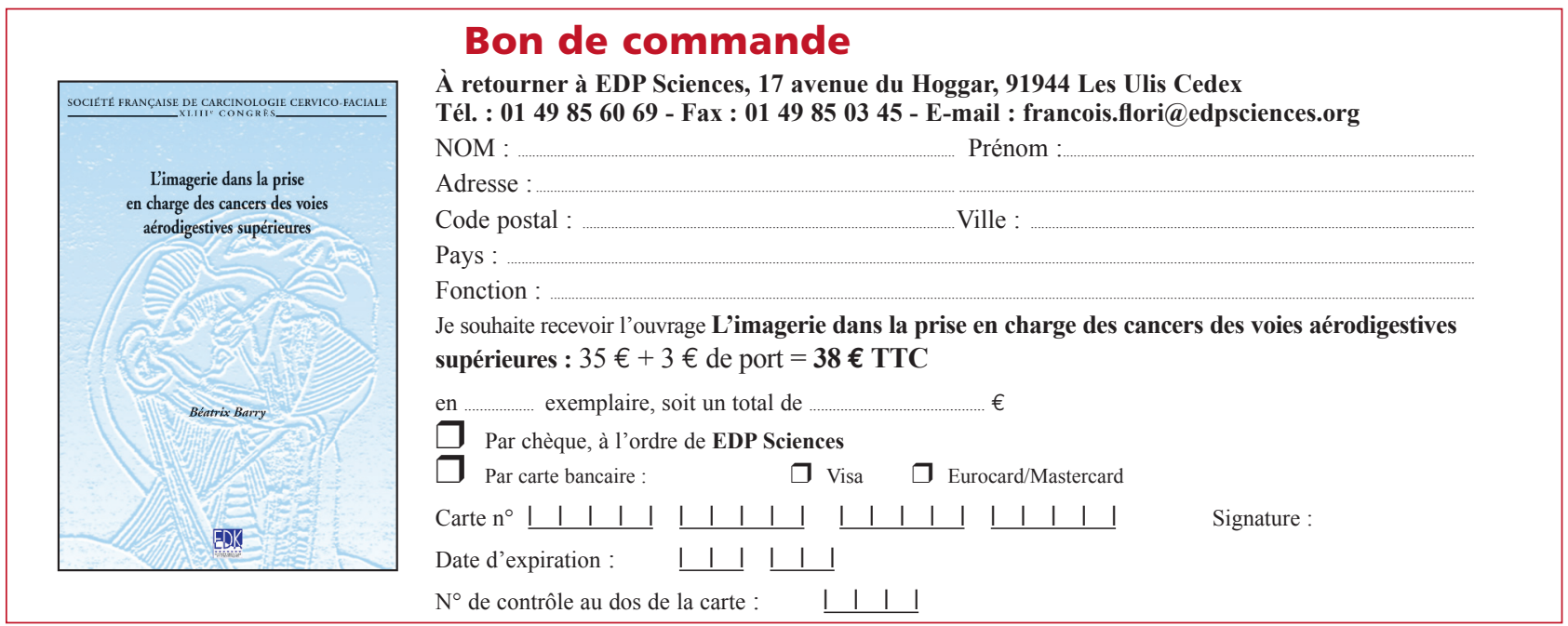

